\title{
Bacteria can exploit a flagellar buckling instability to change direction
}

\author{
Kwangmin Son ${ }^{1}$, Jeffrey S. Guasto ${ }^{2}$ and Roman Stocker ${ }^{2 \star}$
}

\begin{abstract}
Bacteria swim by rotating rigid helical flagella and periodically reorienting to follow environmental cues ${ }^{1,2}$. Despite the crucial role of reorientations, their underlying mechanism has remained unknown for most uni-flagellated bacteria ${ }^{3,4}$. Here, we report that uni-flagellated bacteria turn by exploiting a finely tuned buckling instability of their hook, the 100-nm-long structure at the base of their flagellar filament ${ }^{5}$. Combining high-speed video microscopy and mechanical stability theory, we demonstrate that reorientations occur $10 \mathrm{~ms}$ after the onset of forward swimming, when the hook undergoes compression, and that the associated hydrodynamic load triggers the buckling of the hook. Reducing the load on the hook below the buckling threshold by decreasing the swimming speed results in the suppression of reorientations, consistent with the critical nature of buckling. The mechanism of turning by buckling represents one of the smallest examples in nature of a biological function stemming from controlled mechanical failure $^{6}$ and reveals a new role for flexibility in biological materials, which may inspire new microrobotic solutions in medicine and engineering ${ }^{7}$.
\end{abstract}

Flexibility is woven into every facet of living materials. At the cellular level, flexibility allows red blood cells to squeeze through capillaries $^{8}$ and DNA to stretch and twist to compensate for variability in binding site length ${ }^{9}$. At the organismal level, flexibility enhances structural performance, for example by enabling animal bones and plant branches to absorb mechanical energy ${ }^{10}$. Flexibility also underpins a host of dynamic life functions, including locomotion, reproduction and predation, by enabling the storage and swift release of elastic energy, a mechanism used by jumping froghoppers to escape predators ${ }^{11}$, by plants to catapult seeds for dispersal ${ }^{12}$, and by aquatic invertebrates to suck in prey ${ }^{13}$. An extreme consequence of flexibility is the occurrence of mechanical instabilities, such as buckling and fluttering, which in engineered systems are synonymous with failure ${ }^{14}$, but in natural systems can serve functional purpose. The biomechanical repertoire of organisms includes mechanical instabilities over a wide range of timescales, from the millisecond snap-buckling instabilities that allow Venus flytraps ${ }^{15}$ and humming birds ${ }^{16}$ to capture insects to the gradual buckling responsible for the wavy edges of leaves and flowers ${ }^{17}$.

Flexible appendages are widely used by organisms for locomotion in fluids, from the flapping of bird and bat wings ${ }^{18}$, to the actuation of fish fins ${ }^{19}$, to the bending of sperm flagella ${ }^{20}$. Flexibility also plays a subtle role in the locomotion of bacteria with multiple flagella (peritrichous), such as Escherichia coli, which bundles its flagella together for propulsion $\left(\mathrm{a} \mathrm{run}^{1}\right)$ by exploiting the compliance of the flagellum's base ${ }^{21,22}$. When one or more flagella leave the bundle following a change in the direction of rotation of their motor, the torque resulting from the unbundling, or from the associated polymorphic transformation of the flagellar filament, reorients the cell (a tumble ${ }^{1,21}$ ). This ability to reorient is essential, for example, to climb chemical gradients in search of nutrients or to escape toxins ${ }^{2}$. Yet, many bacteria have only a single flagellum (monotrichous), including 90\% of motile marine bacteria ${ }^{3}$, and how they reorient has long remained unclear. Only recently has the monotrichous marine bacterium Vibrio alginolyticus been shown to exhibit the ability to turn or flick ${ }^{4}$ by a seemingly impossible off-axis motion of its flagellum (Fig. 1a,e). We discovered and experimentally demonstrated that the mechanism responsible for the flick is a buckling instability of the hook, thereby revealing a new, striking role of flexibility in bacterial locomotion and one of the smallest examples in nature of controlled mechanical failure used for biological function.

Detailed observations using high-speed video microscopy revealed a new component of $V$. alginolyticus' swimming pattern (Fig. 1). V. alginolyticus swims in a cyclic pattern, alternating between forward runs, when the flagellum pushes the cell head, and backward runs, when the flagellum pulls the head. At the end of a forward run, the cell reverses, changing its swimming direction by $\Delta \theta \approx 180^{\circ}$. In contrast, cells have recently been reported to reorient by an average angle $\Delta \theta \approx 90^{\circ}$ (a 'flick's) at the end of the backward run and on resuming forward swimming, on the basis of imaging at 30-100 frames s $^{-1}$ (ref. 4). By imaging at up to 1,000 frames s $^{-1}$ (Fig. 1a,b and Supplementary Movies S1-S3), we quantified the cell head and flagellar kinematics during the flick process, discovering that the flick occurs a short period $(\sim 10 \mathrm{~ms})$ after the transition from a backward to a forward run. These dynamics are revealed by the alignment, $q$, between the swimming direction and the cell head orientation (Fig. 1c). Following a backward run $(q=-1)$, the swimming speed drops to zero (Fig. 1d) and within $<1 \mathrm{~ms}$ the cell switches to a forward run $\left(q=1 ; \Delta \theta \approx 180^{\circ}\right.$; Fig. 1c), which lasts $10.2 \pm 5.7 \mathrm{~ms}$ (mean \pm s.d.; $n=52$; Supplementary Fig. S9). Only then, the flick begins, lasting $\sim 60 \mathrm{~ms}$ and characterized by a departure of $q$ from 1.

The discovery of this $\sim 10 \mathrm{~ms}$ delay is essential to correctly assess the state of stress of the cell. During backward swimming, the thrust from the flagellum and the drag on the head are equal in magnitude and directed away from one another ${ }^{21}$, and thus the cell is under tension (Fig. lf). Conversely, during forward swimming it is under compression (Fig. 1g). As structures under compression can lose stability, the occurrence of flicks exclusively during forward swimming led us to reason that their origin lies in a buckling instability of the flagellum, and thus depends on the flagellum's material properties. Recent simulations ${ }^{23}$ have focused on the stability of the flagellar filament, and an instability resulting

${ }^{1}$ Department of Mechanical Engineering, MIT, Cambridge, Massachusetts 02139, USA, ${ }^{2}$ Ralph M. Parsons Laboratory, Department of Civil and Environmental Engineering, MIT, Cambridge, Massachusetts 02139, USA. *e-mail: romans@mit.edu 


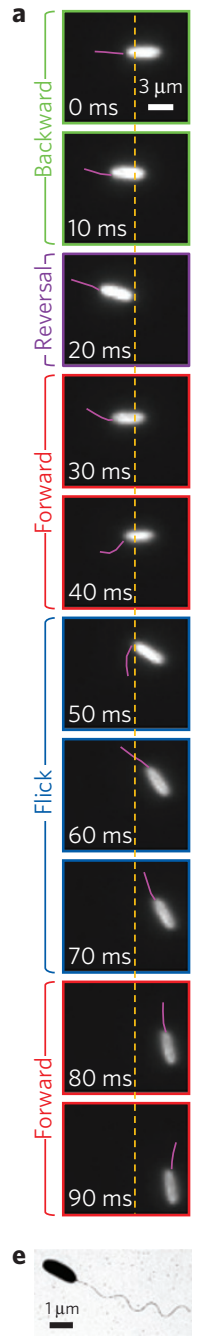

b

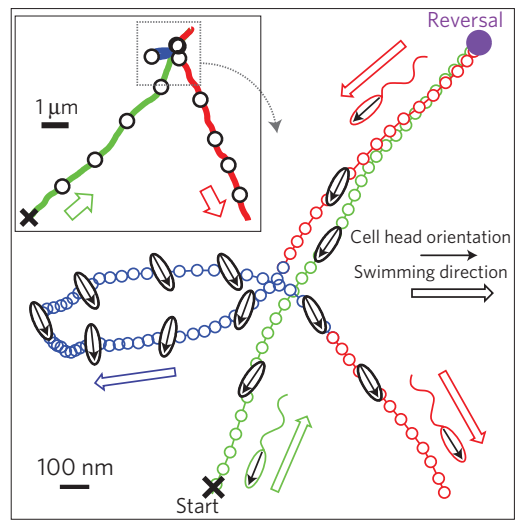

C

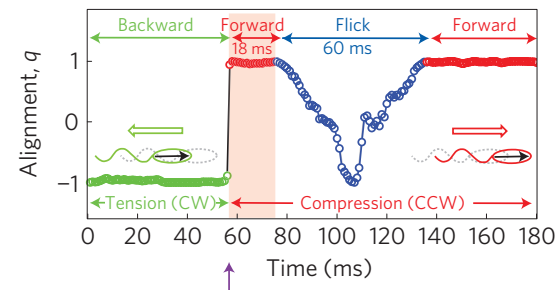

d

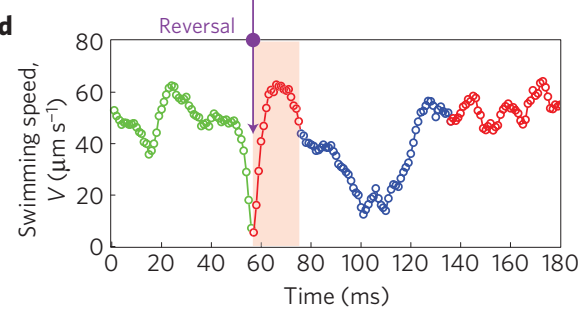

f
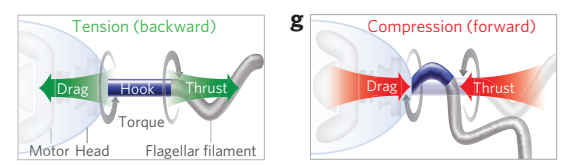

Figure 1 | High-speed video microscopy of V. alginolyticus reveals that flicks occur after the onset of forward swimming. a, Image sequence captured with high-intensity dark-field microscopy at 420 frames s $^{-1}$, showing the kinematics of the cell head and polar flagellum (tracked and coloured magenta; Methods) just before and during a flick (see also Supplementary Movie S1). The dashed orange line provides a reference for cell position. b, Cell trajectory containing a flick, captured with high-speed imaging (1,000 frames s ${ }^{-1}$, phase-contrast microscopy; see also Supplementary Movie S2). Cell head positions are shown by circular markers at $1 \mathrm{~ms}$ intervals. A schematic of the head orientation at selected times is overlaid (not to scale). The inset shows the entire trajectory subsampled at a conventional frame rate of 30 frames s$^{-1}$ (open circles). c) The alignment, $q$ (directional cosine; Supplementary Information S2), between cell head and swimming direction, for the trajectory in $\mathbf{b}$, reveals the distinct elements of the swimming cycle of $V$. alginolyticus, and in particular the short forward swimming segment (here, 18 ms long; red) before the 60-ms-long flick (blue). The inset in $\mathbf{b}$ demonstrates that high-speed imaging is necessary to capture the short delay in the flick. d, Swimming speed during a flick (same colour scheme as in c).

e, Transmission electron micrograph of $V$. alginolyticus, showing the single polar flagellum (mean head length $3.2 \mu \mathrm{m}$, mean flagellar contour length 4.6 $\mu \mathrm{m}$; Supplementary Table S1), which has a sheath that covers it and prevents polymorphic transformations ${ }^{33} . \mathbf{f}, \mathbf{g}$, Schematics (not to scale) of the flagellar filament, hook and rotary motor during backward swimming (f), when the hook is in tension, and during forward swimming ( $\mathbf{g})$, when the hook is in compression. from overcoiling of the flagellum has been suggested as a potential mechanism for the flick ${ }^{4}$. In contrast, we focus on the mechanical stability of the hook, the structure at the base of the flagellum that connects to the motor, because its bending stiffness $E I$ ( $E$ is Young's modulus; $I$ is the area moment of inertia) is 100-1,000 times smaller than that of the flagellar filament (Supplementary Tables S2 and S3), and because most of the deformation during a flick is confined to the base of the flagellum ${ }^{4}$ (Fig. 1a).

The hook of $V$. alginolyticus is a straight, hollow, slender rod (length $L \approx 100 \mathrm{~nm}$ (ref. 5), slenderness ratio $\sim 20$; Supplementary Information S1.2), subject to a typical axial force $F=0.6 \mathrm{pN}$ from the thrust of the flagellum and a typical torque $T=554 \mathrm{pN} \mathrm{nm}$ from the motor (Supplementary Information S1.1). $F$ and $T$ stem from the drag-based thrust of the cell (Supplementary Information S1.1) and are referred to here as viscous loads, because inertial forces are negligible at the bacterial scale ${ }^{21}$. Euler beam theory ${ }^{14}$ predicts that a slender structure buckles above a critical load under compression when $F>F_{\mathrm{CR}}=\pi^{2} E I / L^{2}$ or under torsion when $T>T_{\mathrm{CR}}=2 \pi E I / L$. Under simultaneous loading, buckling occurs when $F / F_{\mathrm{CR}}+\left(T / T_{\mathrm{CR}}\right)^{2}>1$ (ref. 14). V. alginolyticus exceeds this threshold when its swimming speed surpasses $V=51 \mu \mathrm{ms}^{-1}$ (Supplementary Information S1), which is comparable to the mean swimming speed, $V=47 \mu \mathrm{m} \mathrm{s}^{-1}$, at representative marine sodium concentrations $\left(\left[\mathrm{Na}^{+}\right]=513 \mathrm{mM}\right.$; Fig. 2a), indicating that typical swimming loads are sufficient to cause the hook to buckle.

To demonstrate that cells flick because the viscous load exceeds the hook's buckling threshold, we systematically reduced the load on the cells by decreasing their swimming speed. Whereas the link between a reduction in swimming speed and the disappearance of flicking was established qualitatively in the original observation of this process ${ }^{4}$, here a quantification of this relationship enabled the determination of the mechanism underpinning flicks. This was achieved through a reduction in the sodium concentration of the suspending medium, $\left[\mathrm{Na}^{+}\right]$(Fig. 2a; Methods; ref. 4), exploiting the fact that the motor of $V$. alginolyticus is driven by trans-membrane sodium gradients ${ }^{24}$. At $\left[\mathrm{Na}^{+}\right]=100 \mathrm{mM}$ cells swam at $40 \mu \mathrm{m} \mathrm{s}^{-1}$ on average and regularly alternated between flicks and reversals (Fig. 2a and Supplementary Movie S4). In contrast, at $\left[\mathrm{Na}^{+}\right]=3 \mathrm{mM}$ their mean speed fell to $12 \mu \mathrm{m} \mathrm{s}^{-1}$ and flicks nearly disappeared (Fig. 2a and Supplementary Movie S4). To quantify the dependence of flicking on the swimming speed, and thus on the viscous load, we identified all reorientation events (flicks and reversals) from 17,061 trajectories (Supplementary Information S2) recorded over a range of sodium concentrations (Fig. 2b) and computed the probability of flicking during a swimming cycle, $P_{\mathrm{F}}$ (Supplementary Information S3 and S4), as a function of $V$ (Fig. 3a). We found that $P_{\mathrm{F}}$ plummeted from $80 \%$ at speeds $V>47 \mu \mathrm{m} \mathrm{s}^{-1}$ to $10 \%$ at speeds $V<25 \mu \mathrm{m} \mathrm{s}^{-1}$. The steep decrease in $P_{\mathrm{F}}$ when the swimming speed drops below $47 \mu \mathrm{m} \mathrm{s}^{-1}$ is consistent with the critical nature of buckling and with the predicted buckling load of the hook.

If flicks are caused by buckling, how can bacteria achieve steady forward swimming after a flick and suppress further buckling? Their swimming speed, and hence the load, is not significantly different just before a flick compared to a steady run (Fig. 1d and Supplementary Information S6.1). Rather, we find through direct measurements of the flagellar motion that a dynamic stiffening of the hook occurs during swimming (Supplementary Information S5 and Movies S5 and S6). The hook's bending stiffness increases sixfold under the load of steady swimming $\left(E I_{\text {LOADED }}=2.2 \pm 0.4 \times 10^{-25} \mathrm{~N} \mathrm{~m}^{2}\right)$ compared with the unloaded state $\left(E I_{\text {RELAXED }}=3.6 \pm 0.4 \times 10^{-26} \mathrm{~N} \mathrm{~m}^{2}\right)$. This stiffening averts buckling during steady swimming and is probably caused by twisting of the hook, which is known to occur under the motor's torsional load ${ }^{25,26}$. We infer that, on motor reversal, the hook temporarily unwinds, losing bending stiffness and becoming susceptible to buckling (Supplementary Information S7). 

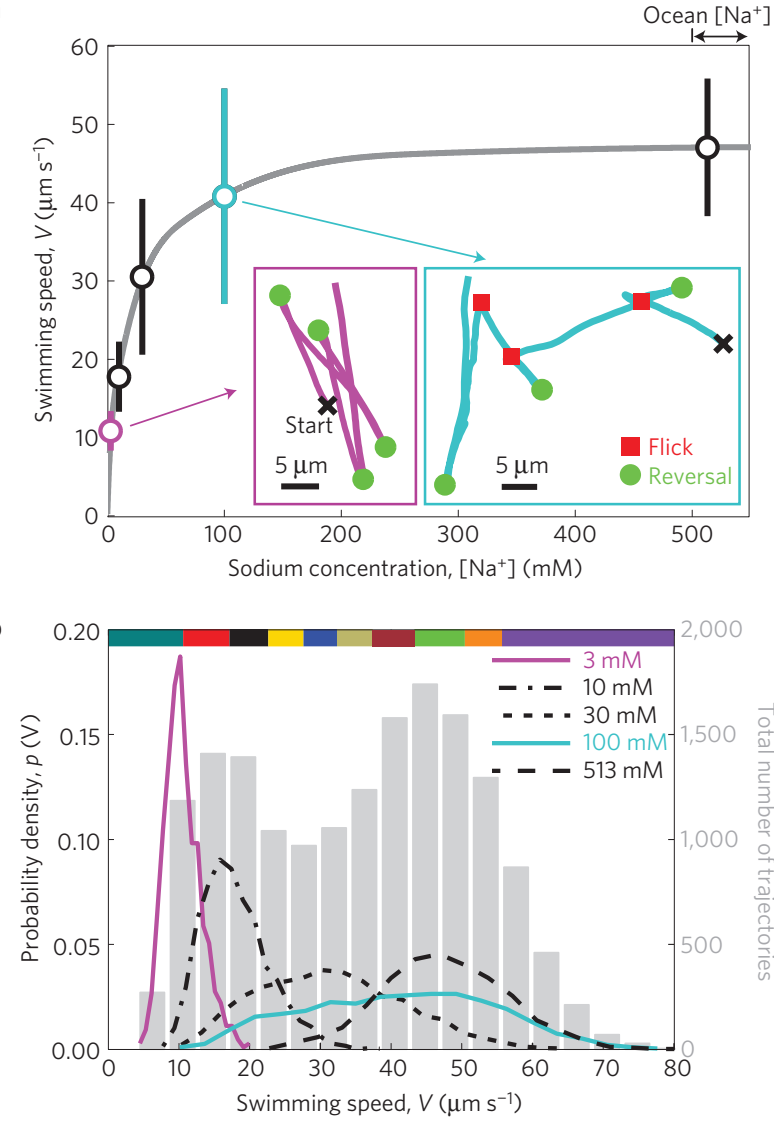

Figure 2 | Reorientation dynamics depend on swimming speed. $\mathbf{a}$, The mean swimming speed of $V$. alginolyticus decreases with the ambient sodium concentration, $\left[\mathrm{Na}^{+}\right]$, allowing for a controlled reduction of the viscous load on cells, which depends linearly on swimming speed ${ }^{21}$. For each sodium concentration, $>2,000$ trajectories were analysed (error bars are standard deviations). Insets show typical trajectories at high ([ $\left.\mathrm{Na}^{+}\right]=100 \mathrm{mM}$, aqua) and low $\left(\left[\mathrm{Na}^{+}\right]=3 \mathrm{mM}\right.$, magenta) sodium concentrations, illustrating the suppression of flicks at low swimming speeds (see also Supplementary Movie S4). b, Probability density function of $V$ (left axis), showing the broad distribution of speeds in a population for any given sodium concentration. The grey histogram denotes the total number of trajectories analysed for each swimming speed (right axis), captured over a range of sodium concentrations $\left(\left[\mathrm{Na}^{+}\right]=3-513 \mathrm{mM}\right)$. Coloured rectangles on the top axis represent speed ranges used in Fig. 3 and include $>1,000$ trajectories each.

Stiffening of the hook under an applied load is in line with crystallographic and electron cryomicroscopy studies in Salmonella typhimurium, which suggest that the hook's interlocking protein microstructure increases its structural stability under torsion ${ }^{27,28}$. Furthermore, optical tweezer measurements of tethered E. coli and Streptococcus show a torsionally soft phase up to $\sim 180^{\circ}$ followed by a torsionally rigid phase for larger deformations, when the hook stiffens torsionally following twisting ${ }^{25,26}$. Ultimately, however, understanding the precise origin of the enhanced stiffness during steady swimming will probably require detailed molecular dynamics simulations of the hook of $V$. alginolyticus.

Buckling during a flick and the subsequent stability during steady forward swimming can both be understood in terms of the hook's stability diagram. The marginal stability condition, $F / F_{\mathrm{CR}}+\left(T / T_{\mathrm{CR}}\right)^{2}=1$, defines a parabolic separatrix in the space of the normalized force, $F / F_{\mathrm{CR}}$, and torque, $T / T_{\mathrm{CR}}$ (Fig. $3 \mathrm{~b}$ ). Inside the parabola, the hook is stable and functional for swimming, whereas outside it buckles and triggers a reorientation. During a

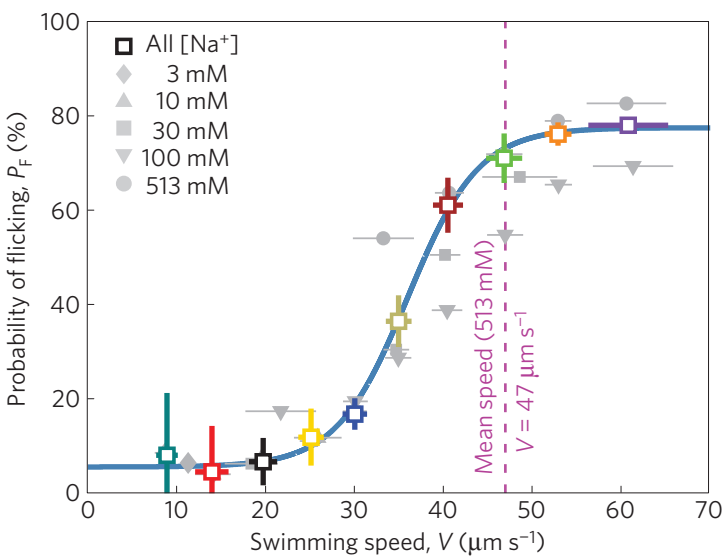

b

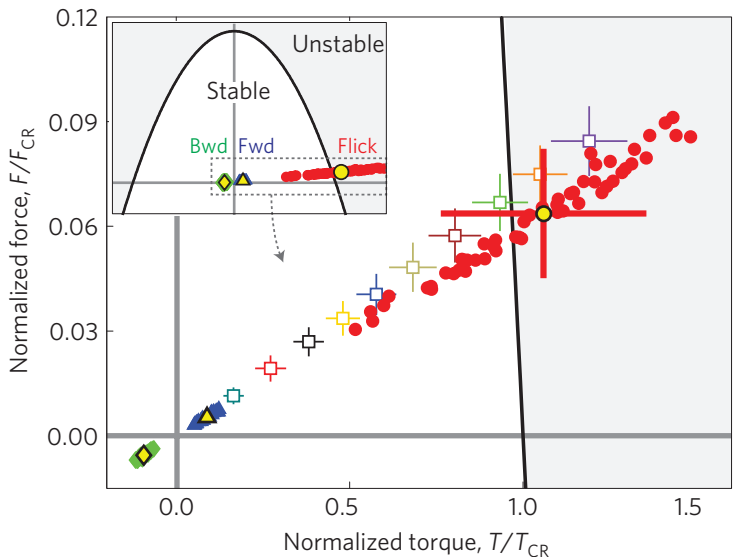

Figure 3 | The probability of flicking shows a sharp increase with increasing load on the hook. $\mathbf{a}$, The probability $P_{F}$ that a cell flicks during a swimming cycle (the sequence of two runs and the two intervening reorientations) increases sharply with swimming speed $V$ (open squares). Cell trajectories for all sodium concentrations, $\left[\mathrm{Na}^{+}\right]$, from Fig. $2 \mathrm{~b}$ are included and binned by swimming speed, with colours corresponding to the speed ranges in Fig. 2b. Cells that flick after the start of each forward run have $P_{\mathrm{F}}=100 \%$, whereas cells that never flick have $P_{\mathrm{F}}=0 \%$. The dashed magenta line indicates the mean swimming speed, $V=47 \mu \mathrm{m} \mathrm{s}^{-1}$, for cells swimming at $\left[\mathrm{Na}^{+}\right]=513 \mathrm{mM}$, representative of natural marine conditions. The grey curve is a logistic fit, and horizontal and vertical error bars denote the standard deviation in cell speed and the error in classifying reorientations, respectively (Supplementary Information S4). The dependence of $P_{\mathrm{F}}$ on $V$ was confirmed by measuring $P_{\mathrm{F}}$ for each individual $\left[\mathrm{Na}^{+}\right]$tested and binning each set by swimming speed (filled grey symbols, $>300$ trajectories each) by exploiting the natural variability in cell speed within a population. This rules out physiological effects associated with changes in $\left[\mathrm{Na}^{+}\right]$as a possible cause of the suppression of flicks. b. Stability diagram of the hook under combined axial and torsional loads. The hook is stable when the normalized viscous force $\left(F / F_{C R}\right)$ and torque $\left(T / T_{C R}\right)$ fall underneath the stability boundary (black curve) and is predicted to buckle otherwise. Symbols represent measurements for steady backward swimming (green diamonds), forward swimming (blue triangles), and short forward runs before flicks (red circles) from 135 trajectories recorded at 1,000 frames $\mathrm{s}^{-1}$ at a sodium concentration of $\left[\mathrm{Na}^{+}\right]=513 \mathrm{mM}$ (Supplementary Information S6.1). Yellow filled symbols represent averages of the three cases and error bars denote standard deviations. Critical loads were computed on the basis of the bending stiffness for the loaded hook during steady swimming and for the relaxed hook during a flick (Supplementary Information S5). Open squares extend the same analysis to the data set from $\mathbf{a}$, demonstrating the loss of stability as swimming speed increases. 

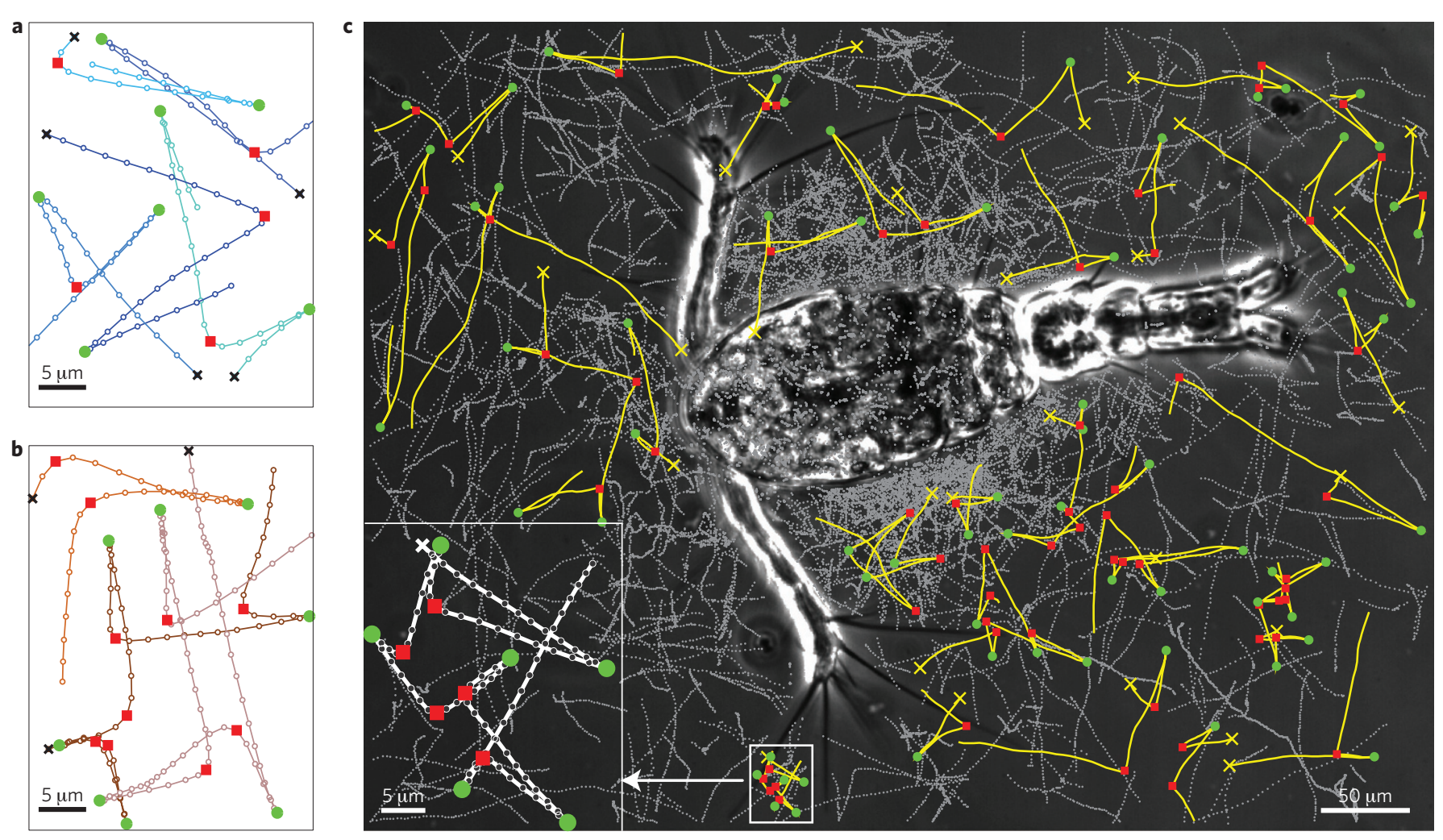

Figure 4 | Turning by buckling is widespread among uni-flagellated bacteria. a-c, Swimming trajectories of $P$. haloplanktis (a), V. coralliilyticus (b) and a natural bacterial community enriched from a seawater sample and exposed to a dead copepod (c; Supplementary Information S8). In all cases, trajectories exhibit the same alternation between $180^{\circ}$ reversals (green circles) and large reorientations having a broad angular distribution centred about $90^{\circ}$ (flicks; red squares) as for V. alginolyticus. Crosses are trajectory starting points and open circles denote cell head positions at 33 ms intervals. Yellow trajectories in c are also captured at $33 \mathrm{~ms}$ intervals and the white inset shows a magnified trajectory.

steady forward and backward swimming, the normalized loads fall within the stability region owing to the higher bending stiffness (higher $F_{\mathrm{CR}}$ and $T_{\mathrm{CR}}$ ). In contrast, just after the onset of forward swimming, immediately following a reversal, the normalized loads fall mostly outside the separatrix owing to the lower bending stiffness, corresponding to buckling and resulting in flicks.

Turning by buckling is a fundamental component of the motility strategy of $V$. alginolyticus, where it occurs in $\sim 80 \%$ of swimming cycles for mean swimming speeds at natural sodium concentrations (Fig. 3a). The observation that $90 \%$ of motile marine bacteria are monotrichous ${ }^{3}$ suggests that the same motility pattern, and potentially the same material properties of the hook, may be widespread among marine bacteria. This hypothesis is supported by our observations, which revealed the same alternation of reversals and flicks in the fast-swimming marine bacterium Pseudoalteromonas haloplanktis ${ }^{29} \quad\left(P_{\mathrm{F}}=40 \%\right.$; Fig. 4a; ref. 4), in the coral pathogen V. coralliilyticus $^{30}\left(P_{\mathrm{F}}=80 \%\right.$; Fig. 4b) and, most strikingly, in a mixed seawater community $\left(P_{\mathrm{F}}=60 \%\right.$; Fig. $4 \mathrm{c}$ and Supplementary Information S8). Other monotrichous motility strategies notwithstanding (for example, the run-and-stop swimming of Rhodobacter sphaeroides ${ }^{21}$ ), turning by buckling may represent a prevalent reorientation mechanism among monotrichous bacteria and a widespread counterpart to the classic tumbling of peritrichous bacteria ${ }^{1}$. Whereas multiple flagella may be justified in nutrient-rich environments to generate the necessary torque to drill through very viscous media ${ }^{31}$, a single flagellum may embody a motility adaptation to oligotrophic environments, such as the ocean ${ }^{4}$, by minimizing the costs of flagellar biosynthesis and actuation ${ }^{32}$. However, this cost-saving strategy introduces the problem of reorientation, for which turning by buckling provides an effective, minimalistic solution.
These findings reveal a new role for flexibility in bacterial locomotion. The highly compliant hook is seemingly the Achilles' heel of $V$. alginolyticus, teetering on the brink of mechanical failure. However, this vulnerability is only engaged when advantageous for the cell to reorient and represents one of the smallest examples in nature of how operating at the boundary of mechanical stability generates new functional solutions. Turning by buckling is an elegant, under-actuated reorientation mechanism that highlights the value of incorporating flexibility and controlled mechanical failure into engineered systems ${ }^{6}$, and enriches the array of biological systems whose extreme mechanics can inspire new robotic solutions in medicine and engineering ${ }^{7}$.

\section{Methods}

Cell culturing. V. alginolyticus YM4 was cultured overnight in VC medium $\left(0.5 \%(\mathrm{w} / \mathrm{v})\right.$ polypeptone, $0.5 \%$ yeast extract, $0.4 \% \mathrm{~K}_{2} \mathrm{HPO}_{4}, 3 \% \mathrm{NaCl}$ and $0.2 \%$ glucose), diluted 1:100 in VPG medium (1\% polypeptone, $0.4 \% \mathrm{~K}_{2} \mathrm{HPO}_{4}, 3 \%$ $\mathrm{NaCl}$ and $0.5 \%$ glycerol $)^{4}$ and grown to late exponential phase $\left(\mathrm{OD}_{600 \mathrm{~nm}}=0.5\right)$. To change the sodium concentration, $\left[\mathrm{Na}^{+}\right]$, cells were washed and resuspended in TMN motility medium ( $50 \mathrm{mM}$ Tris- $\mathrm{HCl}$ ( $\mathrm{pH} 7.5), 5 \mathrm{mM} \mathrm{MgCl}_{2}, 5 \mathrm{mM}$ glucose and $300 \mathrm{mM} \mathrm{NaCl}+\mathrm{KCl}$ ). The difference in $\left[\mathrm{Na}^{+}\right]$was replaced with potassium to maintain osmolarity, a common approach devoid of negative physiological consequences ${ }^{24}$.

Imaging. Flagellar dynamics were visualized in a $25-\mu \mathrm{m}$-deep chamber at 420 frames s $^{-1}$ using high-intensity dark-field microscopy (Nikon Ti-E microscope; $40 \times, 0.6$ NA objective; 1.2 NA oil condenser; mercury lamp illumination) and an Andor Neo camera ( $6.5 \mu \mathrm{m}$ per pixel). To measure cell head dynamics, bacteria were imaged at mid depth in a 120 - $\mu$ m-deep polydimethylsiloxane microchannel at up to 1,000 frames s$^{-1}$ (cell position measurement precision $\sim 20 \mathrm{~nm}$ ) using Photron SA-5 $(20 \mu \mathrm{m} /$ pixel) or SA-3 (17 $\mu \mathrm{m}$ per pixel) high-speed cameras and phase-contrast microscopy $(\times 100,1.4$ NA objective $)$. For the analysis of the probability of flicking and the experiments with varying sodium concentrations, 
cells were imaged at 30 frames s $^{-1}$ by phase-contrast microscopy $(\times 20,0.45$ NA objective) using the Andor Neo camera.

Cell tracking and trajectory analysis. All analyses were performed in Matlab (The Mathworks) using in-house, automated software to track cells, measure cell size and shape, and identify flagellar filaments. Cell trajectories were smoothed using a second-order Savitztky-Golay filter (window sizes: $133 \mathrm{~ms}$ for 30 frames s$^{-1}$, $9.5 \mathrm{~ms}$ for 420 frames s$^{-1}, 4 \mathrm{~ms}$ for 1,000 frames s$^{-1}$ ), and reorientation events were identified using two criteria, a high rate of change of direction and a low instantaneous swimming speed (Supplementary Information S2). Analysis was limited to trajectories containing at least one reorientation, and reorientation events were then classified as flicks or reversals on the basis of the absolute reorientation angle, $\Delta \theta$, defined as the angle between the swimming directions before and after a reorientation (Supplementary Information S3 and S4). Each computer-identified reorientation event was verified manually for 17,061 trajectories before performing statistical analysis.

Received 11 March 2013; accepted 28 May 2013; published online 7 July 2013

\section{References}

1. Berg, H. C. E. coli in Motion (Springer, 2004).

2. Berg, H. C. \& Brown, D. A. Chemotaxis in Escherichia coli analysed by three-dimensional tracking. Nature 239, 500-504 (1972).

3. Leifson, E., Cosenza, B. J., Murchelano, R. \& Cleverdon, R. C. Motile marine bacteria. I. Techniques, ecology, and general characteristics. J. Bact. 87, 652-666 (1964).

4. Xie, L., Altindal, T., Chattopadhyay, S. \& Wu, X. L. Bacterial flagellum as a propeller and as a rudder for efficient chemotaxis. Proc. Natl Acad. Sci. USA 108, 2246-2251 (2011).

5. Koike, M., Terashima, H., Kojima, S. \& Homma, M. Isolation of basal bodies with C-ring components from the $\mathrm{Na}^{+}$-driven flagellar motor of Vibrio alginolyticus. J. Bact. 192, 375-378 (2010).

6. Krieger, K. Buckling down. Nature 488, 146-147 (2012).

7. Sitti, M. Miniature devices: Voyage of the microbots. Nature 458, 1121-1122 (2009).

8. Li, J., Lykotrafitis, G., Dao, M. \& Suresh, S. Cytoskeletal dynamics of human erythrocyte. Proc. Natl Acad. Sci. USA 104, 4937-4942 (2007).

9. Gore, J. et al. DNA overwinds when stretched. Nature 442, 836-839 (2006).

10. Vogel, S. Life's Devices: The Physical World of Animals and Plants (Princeton Univ. Press, 1988).

11. Burrows, M. Biomechanics: Froghopper insects leap to new heights-An innovative leaping action propels these bugs to the top of the insect athletic league. Nature 424, 509-509 (2003).

12. Deegan, R. D. Finessing the fracture energy barrier in ballistic seed dispersal Proc. Natl Acad. Sci. USA 109, 5166-5169 (2012).

13. Singh, A. K., Prabhakar, S. \& Sane, S. P. The biomechanics of fast prey capture in aquatic bladderworts. Biol. Lett. 7, 547-550 (2011).

14. Timoshenko, S. Theory of Elastic Stability 2nd edn (McGraw-Hill, 1961).

15. Forterre, Y., Skotheim, J. M., Dumais, J. \& Mahadevan, L. How the Venus flytrap snaps. Nature 433, 421-425 (2005).

16. Smith, M. L., Yanega, G. M. \& Ruina, A. Elastic instability model of rapid beak closure in hummingbirds. J. Theor. Biol. 282, 41-51 (2011).

17. Sharon, E. et al. Mechanics: Buckling cascades in free sheets-Wavy leaves may not depend only on their genes to make their edges crinkle. Nature 419, 579-579 (2002).
18. Hedenstrom, A., Johansson, L. C. \& Spedding, G. R. Bird or bat: Comparing airframe design and flight performance. Bioinspir. Biomim. 4, 015001 (2009).

19. Lauder, G. V. \& Madden, P. G. A. Fish locomotion: Kinematics and hydrodynamics of flexible foil-like fins. Exp. Fluids 43, 641-653 (2007)

20. Gaffney, E. A. et al. Mammalian sperm motility: Observation and theory. Annu. Rev. Fluid Mech. 43, 501-528 (2011).

21. Lauga, E. \& Powers, T. R. The hydrodynamics of swimming microorganisms. Rep. Prog. Phys. 72, 096601 (2009).

22. Brown, M. T. et al. Flagellar hook flexibility is essential for bundle formation in swimming Escherichia coli cells. J. Bact. 194, 3495-3501 (2012).

23. Vogel, R. \& Stark, H. Motor-driven bacterial flagella and buckling instabilities. Eur. Phys. J. E 35, 1-15 (2012).

24. Sowa, Y., Hotta, H., Homma, M. \& Ishijima, A. Torque-speed relationship of the $\mathrm{Na}^{+}$-driven flagellar motor of Vibrio alginolyticus. J. Mol. Biol. 327, 1043-1051 (2003).

25. Block, S. M., Blair, D. F. \& Berg, H. C. Compliance of bacterial flagella measured with optical tweezers. Nature 338, 514-518 (1989).

26. Block, S. M., Blair, D. F. \& Berg, H. C. Compliance of bacterial polyhooks measured with optical tweezers. Cytometry 12, 492-496 (1991).

27. Samatey, F. A. et al. Structure of the bacterial flagellar hook and implication for the molecular universal joint mechanism. Nature 431, 1062-1068 (2004).

28. Fujii, T., Kato, T. \& Namba, K. Specific arrangement of alpha-helical coiled coils in the core domain of the bacterial flagellar hook for the universal joint function. Structure 17, 1485-1493 (2009).

29. Stocker, R. et al. Rapid chemotactic response enables marine bacteria to exploit ephemeral microscale nutrient patches. Proc. Natl Acad. Sci. USA 105, 4209-4214 (2008).

30. Rosenberg, E. et al. The role of microorganisms in coral health, disease and evolution. Nature Rev. Microbiol. 5, 355-362 (2007).

31. Darnton, N. C., Turner, L., Rojevsky, S. \& Berg, H. C. On torque and tumbling in swimming Escherichia coli. J. Bact. 189, 1756-1764 (2007).

32. Stocker, R. Marine microbes see a sea of gradients. Science 338, 628-633 (2012)

33. Goto, T. et al. A fluid-dynamic interpretation of the asymmetric motion of singly flagellated bacteria swimming close to a boundary. Biophys. J. 89, 3771-3779 (2005).

\section{Acknowledgements}

We thank H. C. Berg, K. Bertoldi, V. Fernandez, K. A. Johnson, A. Lazarus, J. T. Miller, K. Namba, M. Porfiri, T. R. Powers, P. M. Reis and R. Rusconi for for stimulating discussions and valuable feedback, X. L. Wu for supplying Vibrio alginolyticus, S. Smriga for help with seawater samples, Y. Yawata for assistance with cell cultures, and J. Kim for illustrations (Fig. 1f,g). We acknowledge support by a Samsung Scholarship (to K.S.) and NSF grants OCE-0744641-CAREER, IOS-1120200, CBET-0966000 and CBET-1066566 (to R.S.).

\section{Author contributions}

K.S. and J.S.G. performed experiments and analysed data. All authors designed experiments, discussed results and wrote the paper.

\section{Additional information}

Supplementary information is available in the online version of the paper. Reprints and permissions information is available online at www.nature.com/reprints. Correspondence and requests for materials should be addressed to R.S.

\section{Competing financial interests}

The authors declare no competing financial interests. 